\title{
CORRELATION BETWEEN PROLACTIN AND SYMPTOM PROFILE IN ACUTE ADMITTED WOMEN WITH RECURRENT SCHIZOPHRENIA
}

\author{
Miroslav Herceg ${ }^{1,2}$, Krešimir Puljić ${ }^{2}$ Andrea Jambrošić Sakoman ${ }^{2}$, Jelena Sušac ${ }^{2}$, \\ Ivana Todorić Laidlaw ${ }^{2}$, Dora Herceg ${ }^{1} \&$ Mirna Sisek-Šsrem ${ }^{2}$ \\ ${ }^{1}$ School of Medicine, University of Zagreb, Zagreb, Croatia \\ ${ }^{2}$ University Psychiatric Hospital Vrapče, Zagreb, Croatia
}

received: 20.3.2020;

revised: 17.7.2020;

accepted: 7.8.2020

\section{SUMMARY}

Background: The prevalence of hyperprolactinemia among psychiatric patients receiving antipsychotic medications was estimated to be between $30 \%$ and $70 \%$. A review of the literature on prolactin and schizophrenia symptoms suggests that the correlation between them is complex and not limited to the adverse effects of antipsychotics. Relations with specific symptom dimensions have not been found consistently across studies. The association between increased prolactin and recurrent episodes of schizophrenia needs to be replicated in larger samples and in a population of female patients. The aim of this study was to find out whether elevated prolactin is related to specific symptoms or dimensions of schizophrenia, which is a heterogenic entity.

Subjects and methods: The sample consisted of 119 consecutively acute admitted women, aged 18 to 45 years with recurrent schizophrenia diagnosed on bases of DSM-5 criteria. Assessment for all the enrolled subjects comprised a psychiatric evaluation and blood draw to determine the prolactin level. Symptoms of schizophrenia were determined using the Positive and Negative Syndrome Scale (PANSS). Data were analyzed by regression analysis and the Independent Samples $t$ Test. Values are given as means $\pm S D$.

Results: Hyperprolactinemia was detected in $74.79 \%$ patients $(n=89)$, whereas the group without hyperprolactinemia comprised $25.21 \%$ of the sample. When plasma prolactin levels and clinical features between groups were compared, there was a statistically significant difference in the negative subscale scores of the PANSS ( $p=0.0011)$, positive subscale scores of the PANNS $(p=0.0043)$, general subscale scores of the PANSS ( $p=0.0226)$ and total scores of the PANNS $(p=0.0003)$.

Conclusion: There were statistically significant differences in the clinical symptoms between two compared groups in total score and in the positive, negative and general subscores.

Key words: schizophrenia - women-prolactin - psychopathology

$* * * * *$

\section{INTRODUCTION}

Prolactin is a polypeptide hormone secreted by the anterior pituitary gland. Prolactin has multiple functions, including lactation and maternal-infant bonding, in mammals. Numerous factors, including gender, sexual activity, childbirth, stress, smoking, and drugs, can cause the release of prolactin (Kruger et al. 2012). The production of prolactin is inhibited by the release of dopamine in the hypothalamo-pituitary circuit and can be increased by blocking type 2 (D2) dopamine receptors. Most available antipsychotic drugs can therefore cause elevations in prolactin secretion (Haddad \& Wieck 2004, Bostwick et al. 2009). Hyperprolactinemia is a frequent but neglected adverse effect observed in patients treated with antipsychotic-drugs. The prevalence of hyperprolactinemia among psychiatric patients receiving antipsychotic medications was estimated to be between $30 \%$ and $70 \%$ in the western populations and in Japan (Bushe et al. 2008, Kikuchi et al. 2012, Kinon et. al. 2003, Meaney et al. 2004, Montgomery et al. 2004, Smith et al. 2002). An English study showed that $18 \%$ of men and $47 \%$ of women treated with antipsychotics for severe mental illness had a prolactin level above the normal range (Besnard et al. 2014). Hyperprolactinemia is in fact more frequent in women than in men. Sometimes it is asymptomatic, but the higher the prolactin level is, the more patients have clinical manifestations. A review of the literature on prolactin and schizophrenia symptoms suggests that the correlation between them is complex and not limited to the adverse effects of antipsychotics (Rajakumar 2014). The excess mortality in schizophrenia is still a phenomenon insufficiently studied on the cross-national level (Piotrowski et al. 2017). The next question to be considered is whether elevated prolactin is related to specific symptoms or dimensions of schizophrenia, which is a multidimensional entity. Relations with specific symptom dimensions have not been found consistently across studies. We do not know how well prolactin correlates with dopamine activity in the mesolimbic pathway (Rajkumar 2014).

Some of the stuides conducted so far have been exploring the potential strenght of prolactin as a biomarker of antipsychotic response, schizophrenia symptomatology, and tardive dyskinesia, also with inconsistent findings (Souza et al. 2011). 
In regards to the correlation of prolactin level and schizophrenia symptoms some studies have shown a that lower prolactin level is related to positive symptoms: Segal et al. (2007) find significantly lower prolactin levels in male patients with the paranoid subtype of schizophrenia. Segal et al. (2004) also find significantly lower prolactin levels in paranoid schizophrenia compared to schizoaffective and disorganized subtypes. Furthermore, Rinieris et al. (1985) find lower prolactin levels associated with delusions in male patients with schizophrenia. Finally, Johnstone et al. (1977) find serum prolactin negatively correlated with both speech incoherence and total positive symptoms in male patients with chronic schizophrenia. Some other studies have shown that higher prolactin level is associated with negative symptoms: Newcomer et al. (1992) and Akhondzadeh et al. (2006) both find a significant positive correlation between prolactin levels and negative symptoms. On the other hand, some studies have shown that higher prolactin level is associated with positive symptoms: Segal et al. (2007) find a significantly greater elevation of prolactin in male patients with the paranoid subtype of schizophrenia and Prasad (1986) finds higher prolactin levels in the group with positive symptoms. Finally, one study (Luchins et al. 1984) shows no correlation between prolactin levels and psychopathology. The studies above were mainly conducted on male patients, with a sample size of 13 to 57 patients. The examination of a correlation between raised prolactin and recurrent episodes of schizophrenia needs to be replicated in larger samples and in population of female patients. In our own previous research (Herceg et al. 2017) on the influence of hormonal status (estrogen, progesterone and testosterone) and menstrual cycle phase on psychopathology in acute admitted patients with schizophrenia on a sample of 31 patients, no statistically significant differences were found in psychopathology measured by PANSS scale and PANSS subscale (Kay et al. 1987) at the time of admission.

Studies conducted so far have yielded inconstitent results regarding prolactin level and correlation between psychopathology in schizophrenia. The aim of this study was to find out whether elevated prolactin is related to specific symptoms or dimensions of schizophrenia, which is a heterogenic entity.

\section{SUBJECTS AND METHODS}

The sample consisted of 119 consecutively acute admitted women, aged 18 to 45 years with recurrent schizophrenia diagnosed on bases DSM-5 criteria (APA 2013)

All patients were admitted to the acute ward for female psychotic disorders et the University Psychiatric Hospital Vrapče in Zagreb. The sample consisted of women of reproductive age who were regularly menstruating and with regular hormonal activity. Women were excluded if they were taking any synthetic steroids including oral contraceptive, if they were pregnant, lactating or postmenopausal. All patients gave written informed consent, and the University Psychiatric Hospital Vrapče Ethics Committee approved the study. Assessment for all the enrolled subjects comprised of psychiatric evaluation and blood draw for the prolactin level. Prolactin serum samples were assessed on the morning after the admission in 119 recurrent medicated schizophrenia patients. Hyperprolactinemia was defined as a level of prolactin above the upper limit of normal ( $>23.00 \mu \mathrm{g} / \mathrm{L}$ for females). All of the patients were divided into two groups, according to the level of prolactin, that is with and without hyperprolactinemia, respectively. Symptoms of schizophrenia were assessed using Positive and Negative Syndrome Scale (PANSS). The evaluation of clinical status and PANSS was conducted by an experienced psychiatrist blind with regard to the serum prolactin level. Data were analyzed by regression analysis and t-test for independent samples. Values are given as means $\pm \mathrm{SD}$. This research will explore the association between prolactin and psychopathology in female recurrent schizophrenic patients.

\section{RESULTS}

The sample consisted of 119 consecutively acute admitted women, aged 18 to 45 years (mean age $36.74 \pm 7.24$ years) with recurrent schizophrenia diagnosed by DSM-5 criteria. The mean duration of the illness was $8.86 \pm 6.60$ years. The mean age at the onset of the illness was $28.07 \pm 6.43$ years (Table 1).

Table 1. Individual variables in admitted women with recurrent schizophrenia

\begin{tabular}{lc}
\hline & All patients $(\mathrm{n}=119)$ \\
\hline Age (years) & $36.74 \pm 7.24$ \\
Duration of illness (years) & $8.86 \pm 6.60$ \\
Age at onset(years) & $28.07 \pm 6.43$ \\
\hline
\end{tabular}

The mean age in the group of patients with hyperprolactinemia was $36.19 \pm 7.01(n=89)$, whereas in the group of patients without hyperprolactinemia the mean age was $38.37 \pm 7.79(n=30)$. Hyperprolactinemia was detected in $74.79 \%$ patients $(n=89)$, whereas the group without hyperprolactinemia comprised $25.21 \%$ of the sample $(n=30)$ (Table 2). We found that the prevalence of hyperprolactinemia among medicated women with recurrent schizophrenia $(n=119)$ was $75 \%$ (mean serum prolactin level $=83.87 \pm 56.17 \mu \mathrm{g} / \mathrm{L}$, median 36.7 ) (Table 3). There were no differences between the group with normal prolactin level and the group with hyperprolactinemia accordingly to age, duration of illness and age at the onset of the illness. When plasma prolactin levels and clinical features between groups 
Table 2. Individual variables in admitted women with and without hyperprolactinemia

\begin{tabular}{lccc}
\hline & Patients with HL $(\mathrm{n}=89)$ & Patients without HL $(\mathrm{n}=30)$ & $\mathrm{p}$-value \\
\hline Age (years) & $36.19 \pm 7.01$ & $38.37 \pm 7.79$ & $0.1557^{*}$ \\
Duration of illness (years) & $8.33 \pm 6.19$ & $10.43 \pm 7.58$ & $0.1310^{*}$ \\
Age at onset (years) & $28.07 \pm 6.13$ & $28.07 \pm 7.37$ & $0.9996^{*}$ \\
\hline
\end{tabular}

*unpaired t-test

Table 3. Serum prolactin level in women with and without hyperprolactinemia

\begin{tabular}{lcc} 
& Patients with HL $(\mathrm{n}=89)$ & Patients without HL $(\mathrm{n}=30)$ \\
\hline Min & 25.0 & 4.5 \\
Max & 282.6 & 22.6 \\
Mean value and standard deviation & $83.87 \pm 56.17$ & $14.47 \pm 4.93$ \\
Median & 36.7 & 17.1 \\
\hline
\end{tabular}

Table 4. Psychopatology at the time of hospital admission in women with and without hyperprolactinemia

\begin{tabular}{lccc} 
& Patients with HL $(\mathrm{n}=89)$ & Patients without HL $(\mathrm{n}=30)$ & $\mathrm{p}$-value \\
\hline PANSS total & $100.18 \pm 11.18$ & $91.90 \pm 8.52$ & $0.0003^{*}$ \\
PANSS positive & $25.61 \pm 3.94$ & $23.33 \pm 2.86$ & $0.0043^{*}$ \\
PANSS negative & $21.67 \pm 5.18$ & $18.13 \pm 4.50$ & $0.0011^{*}$ \\
PANSS general & $52.74 \pm 4.93$ & $50.43 \pm 4.07$ & $0.0226^{*}$ \\
\hline
\end{tabular}

*unpaired t-test

were compared, there was a statistically significant difference in the negative subscale scores of the PANSS $(p=0.0011)$, positive subscale scores of the PANNS $(p=0.0043)$, general subscale scores of the PANSS $(p=0.0226)$ and total scores of the PANNS $(p=0.0003)$ (Table 4). Therefore, we demonstrated a prolactin plasma level effect on general psychiatric symptoms in schizophrenia and, more specifically, on the positive, negative and general subscore on the Positive and Negative Syndrome Scale (PANSS), between two groups at the time of acute admission. There were no statistically significant correlations between level of prolactin and PANNS subscales or PANNS total scores in the entire sample (Table 5).

Table 5. PANSS in correlation with prolactin

\begin{tabular}{ll}
\hline & P-value \\
\hline Positive subscale & $0.5627^{*}$ \\
Negative subscale & $0.7175^{*}$ \\
General subscale & $0.8677^{*}$ \\
Total score & $0.6856^{*}$ \\
\hline *linear regression &
\end{tabular}

\section{DISCUSSION}

This study was conducted to examine the association between prolactin plasma levels and psychopathology measured using Positive and Negative Syndrome Scale (PANSS) in female recurrent schizophrenic patients. Although we have shown that prolactin plasma levels effect on general psychiatric symptoms (on the positive, negative and general subscore of
PANSS as well as total PANSS), between patients with and without hyperprolactinemia between two groups, there was no significant difference in the entire sample. Our findings may support a possible role of hyperprolactinemia in recurrent episodes of schizophrenia in female patients (taking into account the total number of subjects with elevated prolactin levels), but further research is required to confirm these results. The percentage of hyperprolactinemia of $74.79 \%$ in this study can be attributed to the fact that the sample consisted of women with recurrent schizophrenia who were receiving antipsychotic medication for a period of time, we did not specifiy the type of antypsychotic medication. Symptoms related to the endocrine system, specifically to the prolactin level, were not evaluated prior to psychiatric illness. Hyperprolactinemia may be present in patients with schizophrenia independent of antipsychotic medication (Riecher-Rössler et al. 2013). The importance of stress (which can induce hyperprolactinemia) as a risk factor should be emphasized (as enhanced prolactin can increase dopamine release through a feedback mechanism and that could contribute to explaining how stress can trigger the outbreak of psychosis), although some studies found no evidence to support the notion that stress is related to elevated serum prolactin at the onset of psychosis (Lally et al. 2017). It should also be taken into account that the function of hypothalamicpituitary-gonadotropin axis affects the endocrine, reproductive and metabolic systems and that hyperprolactinemia has short and long term repercussions that can seriously affect the quality of life: menstrual disturbances (oligomenorrhoea and amenorrhea), 
galactorrhea, sexual dysfunction, gynecomastia, infertility, decreased bone mineral density and breast cancer (Bostwick et al. 2009). Also, it is important to exclude other causes of prolactin elevation, in particular tumours in the hypothalamic-pituitary area (Haddad \& Wieck 2004). As previously described, some studies have shown that elevated prolactin levels are associated with negative symptoms measured by PANSS, but it should be noted that other psychiatric symptoms such as anxiety, somatization, hostility and depression are associated with hyperprolactinemia (Gomes et al. 2015). The occurrence of negative symptoms in psychosis (especially in first episodes) should be distinguished from the potential atypical clinical presentation of depressive and anxiety disorders (and associated sexual dysfunctions such as decreased libido, delayed orgasm, anorgasmia and sexual arousal difficulties- which can again be related to the level of prolactin) by the use of other psychiatric scales (such as the Calgary Depression Scale for Schizophrenia and Montgomery-Åsberg Depression Rating Scale). The Positive and Negative Syndrome Scale (PANSS) is a standard, ubiquitous and proven method used in research related to psychotic disorders, but it is worth emphasizing that some studies used clustering and factor analysis identified an uncorrelated PANSS score matrix (UPSM) that generated transformed PANSS factor scores with high face validity (good correlation with standard PANSS factors), and high specificity/ orthogonality (Hopkins SC et al. 2018). Perhaps, by such analysis of results obtained by PANSS, more reliable data linking certain symptoms of psychosis with levels of prolactin would be obtained. The association of hyperprolactinemia with the severity of the clinical picture has also been demonstrated, higher prolactin values indicating a stronger clinical picture, which calls into question the protective role of prolactin in psychotic disorders (Vuk Pisk et al. 2019). Taking into account all the above, consideration should be given to the possibility of screening for the prolactin levels before beginning treatment with antipsychotic drugs.

\section{Limitations of the study}

The observation of hyperprolactinemia in people with mental illness is complicated due to the wellknown correlation between antipsychotic medication and hyperprolactinemia. The limitation of our study was due to the fact that previous research showed that ideally antipsychotic treatment should be interrupted for at least 72 hours before taking the blood sample, if it can be performed safely (Casanueva et al. 2006). Since all patients were acutely admitted to the acute ward for female psychotic disorders, their mental condition was to be immediately treated using pharmacotherapy due to ethical principles.

\section{CONCLUSION}

In this study we demonstrated the effect of prolactin plasma level on symptoms of schizophrenia between groups of patients with a normal prolactin level and group with hyperprolactinemia and, more specifically, on the positive, negative and general subscore on the Positive and Negative Syndrome Scale (PANSS) at the time of acute admission. There were no statistically significant differences in the clinical features in the entire sample. There were statistically significant differences in the clinical symptoms between two compared groups in the total score and in the positive, negative and general subscores. This work is part of a larger study of hormonal status in female schizophrenic patients where numerous other hormones were monitored. We found an association between prolactin and aggression from this study (Puljic et al. 2020). To the best of our knowledge, this is the first study which demonstrates a significant association between high aggression and high PRL in female patients with schizophrenia. The importance of the results of this research in the future is to think about the routine determination of prolactin as a biomarker for certain psychiatric symptoms but also the prevention of side effects and early detection of prolactinomas, breast and uterine cancer in the female population suffering from schizophrenia.

Considering all previous results, our research, contradictions between the research and necessary limitations of this study, we hope that these findings might help clarify the heterogeneity of schizophrenia and further personalize pharmacotherapy treatments in the future.

Acknowledgements: None.

Conflict of interest: None to declare.

\section{Contribution of individual authors:}

Miroslav Herceg, Krešimir Puljić \& Dora Herceg contributed to the data collection.

Miroslav Herceg, Andrea Jambrošić Sakoman \& Jelena Sušac substantial contributed to conception and design, revision of the results and making conclusions.

Krešimir Puljić, Andrea Jambrošić Sakoman, Jelena Sušac, Ivana Todorić Laidlaw, Dora Herceg \& Mirna Sisek-Šprem contributed to the literature search and revised the manuscript for important intellectual content and substantially contributed to conception and design.

All of the authors critically reviewed the final draft of the manuscript and gave their final approval of the version to be published. 


\section{References}

1. Akhondzadeh S, Rezaei F, Larijani B, Nejatisafa AA, Kashani L \& Abbasi SH: Correlation between testosterone, gonadotropins and prolactin and severity of negative symptoms in male patients with chronic schizophrenia. Schizophrenia Research 2006; 84:405-410

2. American Psychiatric Association: Diagnostic and statistical manual of mental disorders (5th ed.). Washington, DC: Author, 2013

3. Besnard I, Auclair V, Callery G, Gabriel-Bordenave C\& Roberge C: Antipsychotic-drug-induced hyperprolactinemia: physiopathology, clinical features and guidance. Encephale 2014; 40:86-94

4. Bostwick JR, Guthrie SK \& Ellingrod VL: Antipsychotic induced hyperprolactinemia. Pharmacotherapy 2009; 29:64-73

5. Bushe $C$, Shaw $M \&$ Peveler RC: A review of the association between antipsychotic use and hyperprolactinaemia. J Psychopharmacol 2008; 22:46-55

6. Casanueva FF, Molitch ME, Schlechte JA, Abs R, Bonert $V$, Bronstein MD, Brue T, Cappabianca P, Colao A, Fahlbusch R, Fideleff H, Hadani M, Kelly P, Kleinberg D, Laws E, Marek J, Scanlon M, Sobrinho LG, Wass JA \& Giustina A: Guidelines of the Pituitary Society for the diagnosis and management of prolactinomas. Clin Endocrinol 2006; 65:265-73

7. Gomes J, Sousa A \& Lima G: Hyperprolactinemia: Effect On Mood? European Psychiatry 2015; 30:714

8. Haddad PM \& Wieck A: Antipsychotic-induced hyperprolactinaemia: mechanisms, clinical features and management. Drugs 2004; 64:2291-314

9. Herceg $M$, Puljić $K$, Sisek-Šprem $M$ \& Herceg D: Influence of Hormonal Status and Menstrual Cycle Phase on Psychopatology in Acute Admitted Patients with Schizophrenia. Psychiatr Danub 2018; 30(Suppl 4): 175-179

10. Hopkins SC, Ogirala A, Loebel A \& Koblan KS: Transformed PANSS Factors Intended to Reduce Pseudospecificity Among Symptom Domains and Enhance Understanding of Symptom Change in AntipsychoticTreated Patients With Schizophrenia. Schizophr Bull 2018; 6:593-602

11. Johnstone EC, Crow TJ \& Mashiter K: Anterior pituitary hormone secretion in chronic schizophrenia: an approach to neurohumoral mechanisms. Psychological Medicine 1977; 7:223-8

12. Kay SR, Fiszbein A \&Opler LA: The positive and negative syndrome scale (PANSS) for schizophrenia. Schizophr Bull 1987; 13:261-76

13. Kikuchi, T, Iwamoto, K, Sasada, K, Aleksic B, Yoshida K, $\&$ Ozaki N: Sexual dysfunction and hyperprolactinemia in Japanese schizophrenic patients taking antipsychotics. Progress in NeuroPsychopharmacology \& Biological Psychiatry 2012; 37:26-32

14. Kinon BJ, Gilmore JA, Liu H \& Halbreich UM: Prevalence of hyperprolactinemia in schizophrenic patients treated with conventional antipsychotic medications or risperidone. Psychoneuroendocrinology 2003; 28:55-68

15. Kruger THC, Leeners B, Naegeli E et al.: Prolactin secretory rhythm in women: immediate and long-term alterations after sexual contact. Human Reproduction 2012; 27:1139-43
16. Lally J, Ajnakina O, Stubbs B, Williams HR, Colizzi M, Carra E, Fraietta S, Gardner-Sood P, Greenwood KE, Atakan Z, Mondelli V, Ismail K, Howes O, Taylor DM, Smith S, Hopkins D, Murray RM \& Gaughran F: Hyperprolactinaemia in first episode psychosis - A longitudinal assessment. Schizophr Res 2017; 189:117-25

17. Luchins DJ, Robertson AG \& Meltzer HY: Serum prolactin, psychopathology, and ventricular size in chronic schizophrenia. Psychiatry Research 1984; 12:149-53

18. Meaney AM, Smith S, Howes OD, O'Brien M, Murray RM \& O'Keane $V$ : Effects of long-term prolactin-raising antipsychotic medication on bone mineral density in patients with schizophrenia. British Journal of Psychiatry 2004: 184:503-8

19. Montgomery J, Winterbottom E, Jessani M, Kohegyi E, Fulmer J, Seamonds B \& Josiassen RC: Prevalence of hyperprolactinemia in schizophrenia: Association with typical and atypical antipsychotic treatment. Journal of Clinical Psychiatry 2004; 65:1491-8

20. Newcomer JW, Riney SJ, Vinogradov $S$ \& Csernansky JG: Plasma prolactin and homovanillic acid as markers for psychopathology and abnormal movements during maintenance haloperidol treatment in male patients with schizophrenia. Psychiatry Research 1992; 41:191-202

21. Piotrowski P, Gondek $T$ M, Królicka Deręgowska A, Misiak B, Adamowski $T$ \& Kiejna A: An updated review of European studies. Psychiatr Danub 2017; 29:108-20

22. Prasad AD: Concentration of prolactin in the plasma of schizophrenic patients with positive and negative symptoms. Zhurnal Nevropatologii i Psikhiatrii Imeni S.S. Korsakova 1986; 86:1400-1

23. Puljic K, Herceg M, Tudor L \& Pivac N: The association between prolactin concentration and aggression in female patients with schizophrenia. The World Journal of Biological Psychiatry 2020. doi:10.1080/15622975.2020.1795254

24. Rajkumar PR: Prolactin and Psychopathology in Schizophrenia: A Literature Review and Reappraisal. Schizophr Res Treatment 2014; 2014:175360. doi:10.1155/2014/175360. Epub 2014 Mar 27

25. Riecher-Rössler A, Rybakowski JK, Pflueger MO, Beyrau $R$, Kahn RS, Malik $P$ \& Fleischhacker WW; EUFEST Study Group: Hyperprolactinemia in antipsychotic-naive patients with first-episode psychosis. Psychol Med 2013; 43:2571-82

26. Rinieris $P$, Markianos $M$, Hatzimanolis $J$ \& Stefanis $C$ : A psychoendocrine study in male paranoid schizophrenics with delusional ideas of homosexual content. Acta Psychiatrica Scandinavica 1985; 72:309-14

27. Segal M, Avital A, Berstein S, Derevenski A, Sandbank $S$ \& Weizman A: Prolactin and estradiol serum levels in unmedicated male paranoid schizophrenia patients. Progress in Neuro-Psychopharmacology and Biological Psychiatry 2007; 31:378-82

28. Segal M, Avital A, Derevenski A, Berstein S, Sandbank S $\&$ Weizman A: Prolactin serum levels in paranoid versus nonparanoid male schizophrenia patients treated with risperidone. International Clinical Psychopharmacology 2007; 22:192-6

29. Segal M, Avital A, Rojas M, et al.: Serum prolactin levels in unmedicated first-episode and recurrent schizophrenia patients: a possible marker for the disease's subtypes. Psychiatry Research 2004; 127:227-35 
30. Smith S, Wheeler MJ, Murray R \& O'Keane V: The effects of antipsychotic-induced hyperprolactinaemia on the hypothalamic-pituitary-gonadal axis. J Clin Psychopharmacol 2002; 22:109-14

31. Souza RP, Meltzer HY, Lieberman JA, Voineskos AN, Remington $G$ \& Kennedy $J L$ : Prolactin as a biomarker for treatment response and tardive dyskinesia in schizophrenia subjects: old thoughts revisited from a genetic perspective. Hum Psychopharmacol 2011; 26:21-7

32. Vuk Pisk S, Matić K, Gereš N, Ivezić E, Ruljančić $N \&$ Filipčić I: Hyperprolactinemia - side effect or part of the illness. Psychiatr Danub 2019; 31(Suppl 2):148-52

Correspondence:

Miroslav Herceg, MD, PhD

University Psychiatric Hospital Vrapče

Bolnička c. 32, 10000 Zagreb, Croatia

E-mail: miroslav.herceg@bolnica-vrapce.hr 